\title{
A Single Point Mutation in the Plasmodium Falciparum 3'-5 'Exonuclease Does Not Alter Piperaquine Susceptibility
}

\author{
Nonlawat Boonyalai ( $\nabla$ nonlawatb.fsn@afrims.org ) \\ Armed Forces Research Institute of Medical Sciences https://orcid.org/0000-0002-9058-0840 \\ Kirakarn Kirativanich \\ Armed Forces Research Institute of Medical Sciences \\ Chatchadaporn Thamnurak \\ Armed Forces Research Institute of Medical Sciences \\ Chantida Praditpol \\ Armed Forces Research Institute of Medical Sciences \\ Brian A. Vesely \\ Armed Forces Research Institute of Medical Sciences \\ Mariusz Wojnarski \\ Armed Forces Research Institute of Medical Sciences \\ John S. Greisenbeck \\ Armed Forces Research Institute of Medical Sciences \\ Norman C. Waters \\ Armed Forces Research Institute of Medical Sciences
}

\section{Research Article}

Keywords: Exonuclease, Gene Editing, Malaria, Piperaquine resistance, Plasmodium falciparum

Posted Date: January 19th, 2022

DOI: https://doi.org/10.21203/rs.3.rs-1228953/v1

License: (c) (i) This work is licensed under a Creative Commons Attribution 4.0 International License. Read Full License 


\section{Abstract \\ Background}

The rise in Plasmodium falciparum resistance to dihydroartemisinin-piperaquine (DHA-PPQ) treatment has been documented in the Greater Mekong Subregion with associations with mutations in the $P$. falciparum chloroquine resistance transporter ( $p f c r t$ ) and plasmepsin 2 ( $p f p m 2$ ) genes. However, it is unclear whether other genes also play a role with PPQ resistance, such as the E415G mutation in the exonuclease ( $p f e x o$ ) gene. The aim of this study was to investigate the role of this mutation in PPQ resistance by generating transgenic parasites expressing the pfexo-E415G mutant allele.

\section{Methods}

Transgenic parasite clones carrying the E415G mutation in pfexo of the B5 isolate were derived by CRISPR-Cas 9 gene editing and verified using PCR and gene sequencing. Polymorphisms of pfkelch-13, pfcrt, and pfexo were examined by PCR while the copy number variations of $p f p m 2$ were examined by both relative quantitative real-time PCR and the duplication breakpoint assay. Drug sensitivity against a panel of antimalarials, the ring-stage survival assay (RSA), the PPQ survival assay (PSA), and bimodal dose-response curves were used to evaluate antimalarial susceptibility.

\section{Results}

The transgenic line, B5-rexo-E415G-B8, was successfully generated. The PPQ-IC ${ }_{90}, \% P P Q$ survival, and the bimodal dose-response clearly showed that E415G mutation in PfEXO of B5 isolate remained fully susceptible to PPQ. Furthermore, growth assays demonstrated that the engineered parasites grew slightly faster than the unmodified parental isolates whereas $P$. falciparum isolates harbouring $p f k e l c h-13, p f c r t$, and $p f e x o$ mutations with multiple copies of pfpm2 grew much more slowly.

\section{Conclusions}

Insertion of the E415G mutation in PfEXO did not lead to increased PPQ-IC ${ }_{90}$ and \% PPQ survival, suggesting that this mutation is not associated with PPQ resistance and unsuitable to be used as a molecular marker for monitoring PPQ-resistant parasites. The results also highlight the importance of monitoring and evaluating suspected genetic mutations with regard to parasite fitness and resistance.

\section{Background}

Artemisinin-based combination therapies (ACTs) remain the recommended first-line antimalarial therapy for uncomplicated Plasmodium falciparum infections in almost all endemic countries [1]. ACTs consist of two drugs: a semisynthetic artemisinin (ART) derivative and a longer lasting partner drug. Currently, six ACTs are available including artemether+lumefantrine (AL), artesunate+amodiaquine (AS+AQ), artesunate+mefloquine (AS+MQ), artesunate+sulfadoxine-pyrimethamine (AS+SP), dihydroartemisinin+piperaquine (DHA+PPQ) and most recently artesunate+pyronaridine (AS+PND) [1]. However, the effectiveness of the ACTs is threatened by the emergence of parasites with decreased susceptibility to the ART derivatives and/or resistance to ACT partner drugs [2-4]. Soon after DHA-PPQ was recommended by WHO as a first-line therapy for uncomplicated $P$. falciparum malaria [5], the emergence of DHA-PPQ resistance was reported within the Greater Mekong sub region including Cambodia [6-9], Vietnam [10] and more recently in Thailand [11].

ART resistance is characterized by the presence of mutations in the Pfkelch13 (pfk13) propeller domain which is associated with delayed in vivo parasite clearance times [2,3] and with increased survival in the in vitro ring-stage survival assay (RSA) [12]. There are three molecular marker candidates for PPQ resistance: multiple copies of $P$. falciparum plasmepsin 2 (pfpm2) (PF3D7_1408000) [13-16], novel mutations in $P$. falciparum chloroquine resistance transporter (PfCRT) (PF3D7_0709000) [17-20], and a single mutation in P. falciparum exonuclease (PfEXO) (PF3D7_1362500) $[13,14,21]$. There have been eight previous publications reporting on the E415G mutation in the pfexo gene [13, 22-28]. In addition to the amplification of pfpm2 and pfpm3, Amato R et al., [13] identified a non-synonymous single nucleotide polymorphism (SNP) E415G substitution on an exonuclease-encoding gene from recrudescence isolates from DHA-PPQ treatment failures in Cambodia. The prevalence of DHA-PPQ treatment failures was at 2\% in Ratanakiri, 16\% in Preah Vihear, and 46\% in Pursat. In Vietnam, neither PfEXO-E415G mutations nor pfpm2 amplification was observed in parasites from south-central Vietnam between 2015 and 2016 [27], but $P$. falciparum samples collected from 2018 to 2019 in Vietnam's Central Highlands [25] were found to harbour the PfEXO-E415G mutation with a prevalence of 66.7\% and 85.5\% in Dak Nong and Dak Lak, respectively. Si, Y et. al. [26] has recently reported that no parasites from the China-Myanmar border carried PfEXO-E415G, novel PfCRT mutations, or pfpm2 amplification, even though some parasites with higher PPQ survival assay (PSA) values were detected, indicating different mechanisms of reduced PPQ susceptibility. In Africa, Robert MG et al., [23] first investigated the presence of 
both the PfEXO-E415G mutation and multiple copies of pfpm2 in Senegalese parasites; however, no isolated parasites carried both molecular markers. A similar trend was observed in Sudan where no PfEXO-E415G mutations were observed [28]. However, in Mali, of 214 P. falciparum samples from Dangassa, two isolates exhibited the PfEXO-E415G mutation [22].

While the implication of multiple copies of pfpm2 and novel PfCRT mutations on PPQ resistance have been elucidated [19, 20, 29], and the PPQresistance phenotype can be observed in the presence of novel PfCRT mutations with the PfEXO-E415G mutation [24], there is still a lack of confirmation as to whether the PfEXO-E415G mutation alone causes PPQ-resistance. To investigate whether the PfEXO-E415G is directly associated with PPQ resistance, in this study, we used CRISPR-Cas9 genome editing to generate $P$. falciparum Cambodian parasites harbouring PfEXO-E415G. The in vitro susceptibility to piperaquine and other frontline antimalarials of these gene-edited parasites was determined and compared to those of validated PPQ-resistant $P$. falciparum clinical isolates of Cambodian origin.

\section{Materials And Methods}

\section{pfexo gene and amino acid prediction}

The nucleotide sequence of pfexo from 3D7 was obtained from PlasmoDB with the accession number PF3D7_1362500. The P. falciparum B5 line was obtained from the cloning of a Cambodian P. falciparum isolate [24]. This isolate is ART- and PPQ-sensitive, but CQ-resistant. The full-length pfexo gene of B5 was amplified from $P$. falciparum B5 genomic DNA using polymerase chain reaction (PCR) with primers PA_exon1 and Screening_3UTR_Rev (Table S1). The encoded amino acid sequences and the nucleotide sequences were aligned using Clustal Omega [30]. The signal peptide region was predicted using Signa-P.5.0 [31] and the InterPro program was used for protein family classification [32].

\section{P. falciparum culture}

Asexual blood-stages of $P$. falciparum were maintained in fresh human erythrocytes $\left(\mathrm{O}^{+}\right)$in RPMI1640 (Sigma-Aldrich, USA) supplemented with $5.94 \mathrm{gL}^{-1} \mathrm{HEPES}, 2.1 \mathrm{gL}^{-1}$ sodium bicarbonate, $0.1 \mathrm{gL}^{-1}$ gentamycin sulphate, $0.5 \%$ (w/v) Albumax Il, $4 \mathrm{gL}^{-1}$ dextrose $0.05 \mathrm{gL}^{-1}$ hypoxanthine, and $10-15 \%$ of human serum (complete medium) [33]. Human blood products (erythrocytes and serum) were obtained from the Thai Red Cross. Culture flasks were gassed with $5 \% \mathrm{CO}_{2}, 5 \% \mathrm{O}_{2}, 90 \% \mathrm{~N}_{2}$ gas and incubated at $37^{\circ} \mathrm{C}$. For synchronisation, mature schizont stage parasites were isolated on cushions of $75 \%(\mathrm{v} / \mathrm{v}$ ) isotonic Percoll cushion (GE Healthcare Life Science) as previously described [34, 35] whereas enrichment for ring stages following invasion was performed using 5\% (w/v) D-sorbitol [36] The final ring cultures were washed and returned to culture or used as required.

\section{Construction of plasmid constructs to genetically modify $P$. falciparum}

PCR amplicons used in plasmid cloning were generated using Fusion, High Fidelity DNA polymerase (New England Biolabs) and purified using Qiagen PCR purification or Qiagen Gel extraction kits. For diagnostic PCR amplification, GoTaq (Promega) DNA master mix was used. All constructed plasmids were sequenced to verify authenticity (Biobasic, Canada). For parasite genomic DNA extraction, total cell pellets were first treated with $0.15 \%$ saponin in PBS for 10 min, then washed with PBS before DNA was extracted using a DNeasy Blood \& Tissue Kit (Qiagen).

\section{Construct for donor plasmids}

A fragment of exo sequence with E415G mutation (1,441 bp) was commercially synthesised (GenScript, USA), comprising a stretch of native $P$. falciparum 3D7 exo sequence (covering residues Lys283, exo intron, and Asn408) followed by a stretch of recodonized exo gene sequence encoding the 3D7 amino acid residues Met409 to Glu514 with the point mutation at residue 415 from Glu to Gly but using a different codon usage, and finally a stretch of native 3D7 exo sequence covering amino acid residues 515 to the stop codon. This fragment was cloned into pUC57 by ECoRV generated pUC57-Exo-E415G plasmid. To generate a donor plasmid to introduce Glu415Glu wild-type, pUC57-Exo-E415E plasmid was derived from pUC57-Exo-E415G by Q5 site-directed mutagenesis (New England Biolabs) to alter the codon encoding at amino acid position 415 from GGC to GAA primers Q5SDM_G415E_F and Q5SDM_G415E_R were used (Table S1).

\section{Construct for CRISPR-Cas9 plasmids}

pDC2-Cas9-hDHFR-yFCU, containing a Cas9 expression cassette and the drug selection marker human dihydrofolate reductase (hdhfr) [37] was used as a template vector to generate pDC2-Cas9-bsd-yFCU. Since the $P$. falciparum B5 strain is resistant to pyrimethamine, the hdhfr gene was replaced with the blasticidin gene ( $b s d)$. The $b s d$ gene (399 bp) was amplified from PkpSKIP_Pk47 plasmid [38] using primers bsd_Ncol_F and bsd_Sacll_R (Table S1) and was cloned into the Ncol/Sacll-digested pDC2-Cas9-hDHFRyFCU, giving rise to pDC2-Cas9-bsd-yFCU.

Guide RNA sequences specific for targeting at amino acid position 415 were identified using Benchling (https://www.benchling.com/crispr/) (Fig. S1). A pair of complementary oligonucleotides (sgE415G-1F and sgE415G-1R) corresponding to the 19 nucleotides adjacent to the identified PAM sequences were phosphorylated using T4 polynucleotide kinase, annealed and ligated into pDC2-Cas9-bsd-yFCU predigested with Bbsl, resulting in the guide vector pGuide1.4-bsd. 
The donor plasmid pUC57-Exo-E415G was linearized with Scal prior to electroporation. Percoll-enriched mature schizonts of $P$. falciparum B5 were electroporated with $20 \mu \mathrm{g}$ of pGuide1.4-bsd and $60 \mu \mathrm{g}$ of linearized pUC57-Exo-E415G using either Amaxa P3 primary cell 4D Nucleofector X Kit L (Lonza) or Amaxa Basic Parasite Nucleofector Starter Kit as described [39, 40]. Twenty-four hours post-transfection, the electroporated parasites were treated with $5.45 \mu \mathrm{M}$ blasticidin-S-hydrochloride (Sigma-Aldrich, USA) to select for transfectants harbouring pGuide1.4-bsd before returning the cultures to medium without drug. Detection of the exo-E415G modified locus was carried out by diagnostic PCR using primer pairs ExonI_K283_F and Screen_WT_Rev_V616, ExonI_K283_F and Recodon_R, and Recodon_F and Screen_3'UTR_R. The wild-type pfexo locus was detected by diagnostic PCR using primers ExonI_K283_F and Screen_WT_Rev_V616, with a PCR product of 766 bp. Transgenic parasite clones were obtained by limiting dilution cloning by plating a calculated 0.3 parasite per well ( $200 \mu \mathrm{L}$ and $1 \%$ haematocrit) in flat-bottomed 96 -well microplate wells as described [41]. Wells containing single plaques were identified after 10-14 days using an inverted microscope and the parasites subsequently expanded into round-bottomed wells for further analysis. Transgenic parasite clones were finally checked by diagnostic PCR for integration and modification of the endogenous pfexo ${ }^{\mathrm{E} 415 \mathrm{G}}$ gene. A pfexo ${ }^{\mathrm{E} 415 \mathrm{E}}$ transgenic line expressing the wild-type Glu415 codon

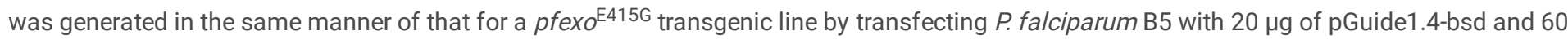
$\mu \mathrm{g}$ of linearized pUC57-Exo-E415E. Once established, all transgenic clones were maintained in medium without any drug.

\section{pfk13, pfcrt, and pfexo genotyping}

Master Cycler Nexus Gradient (Eppendorf) was employed to evaluate the propeller domain of the P. falciparum kelch13 (pfk13) (amino acid residues 442-727) [42, 43], P. falciparum exonuclease (pfexo) SNP at a codon corresponding to the amino acid position 415 [13], and the $P$. falciparum chloroquine resistant transporter (pfcrt) SNPs at codons corresponding to amino acid positions 93, 97, 145, 218, 343, 350, and $353[19,44]$. Primers used to identify pfk13, pfexo, and pfcrt SNPs are shown in Table S1. P. falciparum reference DNAs from 3D7 and W2 clones (Malaria Research \& Reference Reagent Resource, Manassas, VA) were used as positive controls, and all samples were performed in duplicate.

\section{Plasmepsin (pfpm) 1, 2 and 3 Copy number variation assay}

To determine copy numbers of pfpm1 (PF3D7_1407900), pfpm2 (PF3D7_1408000), and pfpm3 (PF3D7_1408100) gene, real-time quantitative PCR (qPCR) was performed on genomic DNA as previously described [29]. The amplification reactions were performed according to Lunaâ Universal qPCR master mix kit (New England Biolabs) with $200 \mathrm{nM}$ of each forward and reverse primer (Table 3 ) and 2 ng of DNA template using Rotor-Gene Q (QIAGEN, Valencia, CA). For the housekeeping gene, b-tubulin (PF3D7_1008700), b-tubulin forward and reverse primers were designed and used as a reference control for all experiments with the same validated PCR conditions as target primers. P. falciparum 3D7 was used as a reference clone. All samples including the references clones were performed in triplicate. The average copy number values for each gene were calculated using $2^{-\Delta \Delta C t}$ method where $\Delta \Delta \mathrm{Ct}$ is [Ct $\left.{ }_{p f p m}-\mathrm{Ct}_{\text {pf } \beta \text {-tubulin }}\right]_{\text {sample }}$ - $\mathrm{Ct}_{\text {pfpm2 }}$ - $\left.\mathrm{Ct}_{\text {pf } \beta \text {-tubulin }}\right]_{3 \mathrm{D} 7}$. Parasites with copy number greater than 1.6 copies for pfpm2 [15] were interpreted to contain multiple copies.

\section{Plasmepsin 2/3 (pfpm2/3) duplication breakpoint PCR assay}

The pfpm2/3 breakpoint PCR assay was performed as previously described [45]. Three pairs of primers (Table 3) were used in this assay. Primers AF_for and AR_rev amplified a 623 bp product surrounding the breakpoint located at the 3' end of pfpm1. Primers BF_for and BR_rev amplified a 484 bp product surrounding the breakpoint at the 3 ' end of pfpm3. Primers BF_for and AR_rev amplified the junction between the breakpoint, producing a $497 \mathrm{bp}$ product in parasite isolates with pfpm2/3 amplifications. A pfpm2/3 single copy isolate is not expected to have the PCR product with these primers. One copy isolate was only noted when the control primer sets amplified a product, and the duplication PCR was negative. Two or more copies were annotated as > 1 copy of pfpm2/3 only when both the control and duplication primer sets generated a product. PCR reactions contained $12.5 \mu \mathrm{L}$ GoTaqÒ Green Master Mix (Promega), $1 \mu \mathrm{L}$ of each primer (10 $\mu \mathrm{M}$ stocks), $3 \mu \mathrm{L}$ of DNA up to $25 \mu \mathrm{L}$ final volume with water. PCR conditions were as follow: $95^{\circ} \mathrm{C}$ for $2 \mathrm{~min}$, followed by 30 cycles of $95^{\circ} \mathrm{C}$ for $45 \mathrm{~s}, 50^{\circ} \mathrm{C}$ for $30 \mathrm{~s}, 72^{\circ} \mathrm{C}$ for $1 \mathrm{~min}$, followed by a 5 -min extension at $72^{\circ} \mathrm{C}$.

\section{In vitro drug susceptibility}

Drug susceptibility testing used HRP-2 ELISA to measure $50 \%$ or $90 \%$ inhibitory concentration ( IC $_{50}$ and IC I0 $\left._{90}\right)$ performed as previously published [46, 47]. In vitro drug susceptibility testing was carried out for control reference clones (W2, D6, C2B) (Malaria Research \& Reference Reagent Resource, Manassas, Vermont, USA) as described previously [48]. $\mathrm{IC}_{50} \mathrm{~S}$ and $\mathrm{IC}_{90} \mathrm{~s}$ were estimated by nonlinear regression analysis using GraphPad Prism version 6.0 program. Samples having poor growth rate, as perceived by obtaining an OD ratio of < 1.7 between the no-drug test wells and the maximum tested drug concentration, were excluded from data analysis.

\section{Bimodal dose response curve}


To determine a bimodal-dose response curve, the PPQ concentration ( 2.44 to $100,000 \mathrm{ng} / \mathrm{mL}$ ) and the dilution series were increased from 8 to 24 points, according to previously published reports $[16,24]$. Culture-adapted clinical isolates or engineered parasites were prepared in the similar manner as in in vitro drug susceptibility testing. The synchronized rings were grown for 72 hours in the presence of different concentrations of PPQ (24-point dilution) in 96-well plates at 1.5\% haematocrit, $0.5 \%$ starting parasitaemia in 0.5\% Albumax RPMI 1640 . Growth at 72 hours was measured by HRP-2 ELISA. Assays were carried out in three biological replicates and the control reference clone W2 was tested along with each culture-adapted clinical isolate. The area under the curve (AUC) for the dose response curve at 0.01 - $100 \mu \mathrm{M}$ was calculated using GraphPad Prism 6.0.

\section{Ring-staged survival assay (RSA)}

In vitro $\mathrm{RSA}_{0-3 \mathrm{~h}}$ was performed on 0-3 h post-invasion rings obtained from selected culture-adapted clinical isolates following published methods [12] with slight modifications. Briefly, parasite cultures were tightly synchronized using 5\% (w/v) D-sorbitol and 75\% Percoll to obtain 0 to 3-h post-invasion rings which were adjusted to $0.5-1 \%$ starting parasitaemia with a $2 \%$ haematocrit in culture media. (0.5\% Albumax RPMI 1640 with 2.5\% AB serum) and cultured in a 48-well microplate with $700 \mathrm{nM} \mathrm{DHA}$ and $0.1 \%$ DMSO in separate wells for growth control. The culture plate was then incubated for 6 hours at $37{ }^{\circ} \mathrm{C}$ in modular incubator chambers and gassed with $5 \% \mathrm{CO}_{2}, 5 \% \mathrm{O}_{2}$ and $90 \% \mathrm{~N}_{2}$ gas. Cells were then washed once, resuspended in drug-free medium, and cultured further for 66 hours. Susceptibility to DHA was assessed microscopically on thin films by estimating the percentage of viable parasites, relative to control (\% survival rate). For the controls, the RSA $\mathrm{A}_{0-3 \mathrm{~h}}$ was also performed on P. falciparum reference clones W2 (ART-sensitive control), IPC-4884 and IPC-5202 (BEI Resources, NIAID, NIH, USA) as ART-resistant control lines. A survival rate $>1 \%$ was deemed resistant for RSA.

\section{Piperaquine survival assay (PSA)}

$\mathrm{PSA}_{0-3 \mathrm{~h}}$ was performed on culture-adapted clinical isolates with 0-3-hour ring-stage parasite cultures following a previously published method [17]. Briefly, parasite cultures were tightly synchronized using 5\% (w/v) D-sorbitol and 75\% Percoll to obtain 0 to 3-hours post-invasion. Synchronized ring parasites at $0.5-1 \%$ starting parasitaemia and $2 \%$ haematocrit were incubated with $200 \mathrm{nM} P P Q$ or $0.5 \%$ lactic acid in water at $37{ }^{\circ} \mathrm{C}$ for 48 hours in a 48-well microplate. The cultures were then washed once, resuspended in drug-free medium, and cultured further for 24 hours. Susceptibility to PPQ was assessed microscopically on thin films by estimating the percentage of viable parasites in the similar manner as RSA. A survival $>10 \%$ was deemed resistant to $P P Q$.

\section{Growth Assays}

For longer-term replication assays, cultures were synchronized as described and resulting in ring stage cultures. The ring stage parasites were synchronized using $5 \%(\mathrm{w} / \mathrm{v})$ D-sorbitol and parasitaemia levels were calculated, and cultures adjusted to $0.1 \%$ parasitaemia, $2 \%$ haematocrit in a final volume of $1 \mathrm{~mL}$ per well of a 12-well plate. Samples were then taken at t $=0,24,72,120$, and $168 \mathrm{~h}$, fixed in $0.8 \%$ glutaraldehyde in PBS and stored at $4^{\circ} \mathrm{C}$ for flow cytometry analysis. Culture media was replaced at 48,71 , and $120 \mathrm{~h}$. Giemsa-stained thin films were also prepared as required for microscopic analysis.

\section{Flow cytometry for parasite quantification}

Parasite samples were fixed in $0.8 \%$ glutaraldehyde in PBS and stored at $4^{\circ} \mathrm{C}$. Cells were prepared for analysis by staining with $2 \mathrm{X}$ SYBR Green I nucleic acid gel stain (Invitrogen, Thermo Fisher Scientific) for $30 \mathrm{~min}$ at $37^{\circ} \mathrm{C}$. Labelling was stopped with an equal volume of PBS and samples acquired using a CytoFlex (Beckman Coulter, USA) with CyEXpert software. Total RBC numbers were calculated using forward- and side-scatter while fluorescence was detected using the 530/630 blue detection laser. Fluorescence intensity was used to distinguish uninfected from infected RBCs, low fluorescence indicating uninfected cells and gating fixed accordingly. Data were analysed using FlowJo.

\section{Statistical analysis}

Statistical analysis was performed using GraphPad Prism version 6.0 (GraphPad Software, Inc., San Diego, CA, USA). The difference of the data between groups was assessed by nonparametric Mann-Whitney test, as appropriate. Statistical significance was defined as a $P$ value of $<0.05$.

\section{Results}

\section{CRISPR-Cas9-mediated editing to introduce pfexo $^{\mathrm{E} 415 \mathrm{G}}$ into $\mathrm{P}$. falciparum parasites}

The pfexo gene from P. falciparum 3D7 (PF3D7_1362500) consists of 2,274 bp with 2 exons and 1 intron. Exon 1 comprises nucleotides 1-949, while the exon 2 starts from nucleotides 1,082-2,274 (Fig. S1). PfEXO consists of 713 amino acids with a molecular mass of 86.7 kDa. The enzyme contains a peptide signal region (residues 1-70) and a 3 ' to $5^{\prime}$ exonuclease domain (residues 445-618). The DNA sequence of the pfexo gene from $P$. falciparum B5 [24], the parental line for genome editing in this study, showed a single non-synonymous mutation, leading to a missense mutation from Lys to Asn at residue 614 (Figs. S2). 
To evaluate the impact of the PfEXO-E415G mutation on parasite susceptibility to piperaquine and other front-line chemotherapies, we used CRISPR-Cas 9 editing to introduce the E415G mutation into the native pfexo gene (Fig. 1A). A pfexo ${ }^{\mathrm{E} 415 \mathrm{G}}$ was efficiently installed onto the $P$. falciparum B5 background [24]. In parallel, a pfexo ${ }^{\mathrm{E} 415 \mathrm{E}}$ transgenic line expressing wild-type E415 codon in the context of recodonization abating the Cas9 protospacer adjacent motif (PAM) site was also attempted to rule out a role for silent mutations in drug susceptibility. Several transfections were performed to introduce both $p f e x o^{\mathrm{E} 415 \mathrm{E}}$ and $p f e x o^{\mathrm{E} 415 \mathrm{G}}$ mutations, but only three transfections provided revived parasites. Two of the three transfections gave rise to the modified pfexo gene, and one transfection did not provide the modified parasites. Successful modification of the pfexo gene in the transfected population following the introduction of the targeting vector was observed around 21 days after transfection and confirmed by diagnostic PCR (Fig. 1B, Pre-cloning). Limiting dilution cloning of the modified parasites resulted in the isolation of parasite clone, B5-rexo-E415G-B8. It is noted that several clones obtained from the cloning did not contain the modified region at pfexo gene. Modification of the native pfexo locus was then confirmed in the transgenic line by diagnostic PCR (Fig. 1B, B5-rexo-E415G-B8) and genomic sequences of transgenic lines (Fig. 1C). Unfortunately, the pfexo $\mathrm{E}^{\mathrm{E} 415 \mathrm{E}}$ transgenic line could not be obtained from this study.

\section{Molecular genotyping of modified pfexo transgenic lines and clinical isolates with PfEXO-E415G mutation.}

The genotypic profiling for piperaquine and artemisinin molecular markers is outlines in Table 1. In addition to the reference clones and engineered parasite lines, two Cambodian isolates from a previous $P$. falciparum clinical efficacy study (ASAP-21 and ASAP-168) [49] were included as they harboured PfEXO-E415G with and without novel PfCRT mutations. As expected, the transgenic B5-rexo-E415G-B8 line carried a similar genotypic profile as the parental B5 line except for the E415G mutation in the pfexo gene. Regarding the two clinical isolates, both carried the PfK13-C580Y and PfEXO-E415G mutations. The ASAP-21 isolate harboured the novel PfCRT-F145I mutation while ASAP-168 did not. Two methods were carried out to determine the copy number variation of the pfpm 2 gene: the SYBR-green qPCR and breakpoint assays [45]. By using the qPCR assay, ASAP-21 was found to contain multiple copies of pfpm2 while ASAP-168 carried a single copy of $p f p m 2$. On the contrary, both ASAP-21 and ASAP-168 isolates were positive for pfpm2/3 amplification as detected by the breakpoint assay (>1 copy) (Fig. S3). The nonconcordant detection between the two methods were also reported by Ansbro, et al [45] and it was suggested that the breakpoint PCR assay is more sensitive than the qPCR assay for detecting minor clones containing the duplication in field isolates. Therefore, in this study, ASAP-21 and ASAP-168 isolates were treated as having multiple copies of pfpm2.

Table 1

Molecular genotyping of pfexo-modified, parental and clinical P. falciparum parasites.

\begin{tabular}{|c|c|c|c|c|c|c|c|c|c|c|c|c|c|}
\hline \multirow[t]{2}{*}{ Sample } & \multirow{2}{*}{$\begin{array}{l}\text { PfK13 } \\
\text { C580Y }\end{array}$} & \multirow{2}{*}{$\begin{array}{l}\text { PfEXO } \\
\text { E415G }\end{array}$} & \multicolumn{7}{|l|}{ PfCRT } & \multicolumn{3}{|c|}{$\begin{array}{l}\text { Copy Number } \\
\text { Variation }\end{array}$} & \multirow{2}{*}{$\begin{array}{l}\text { pfpm } 2 / 3 \\
\text { break } \\
\text { point }\end{array}$} \\
\hline & & & T93S & H97Y & F145I & I218F & M343L & C350R & G353V & pfpm1 & pfpm2 & pfpm3 & \\
\hline $3 \mathrm{D} 7$ & C & $E$ & $\mathrm{~T}$ & $\mathrm{H}$ & $\mathrm{F}$ & I & M & C & G & 1.00 & 1.00 & 1.00 & 1 \\
\hline B5 & C & $E$ & $\mathrm{~T}$ & $\mathrm{H}$ & $\mathrm{F}$ & I & M & C & G & 1.37 & 0.93 & 1.99 & 1 \\
\hline $\begin{array}{l}\text { B5-rexo- } \\
\text { E415G- } \\
\text { B8 }\end{array}$ & C & G & $\mathrm{T}$ & $\mathrm{H}$ & $\mathrm{F}$ & 1 & M & C & G & 1.96 & 0.77 & 2.71 & 1 \\
\hline $\begin{array}{l}\text { ASAP- } \\
21\end{array}$ & Y & G & $\mathrm{T}$ & $\mathrm{H}$ & 1 & 1 & M & C & G & 1.25 & 2.31 & 2.60 & $>1$ \\
\hline $\begin{array}{l}\text { ASAP- } \\
168\end{array}$ & $Y$ & G & $\mathrm{T}$ & $\mathrm{H}$ & $\mathrm{F}$ & I & M & C & G & 1.18 & 1.11 & 1.88 & $>1$ \\
\hline
\end{tabular}

Underline and bold letters indicate either mutations or multiple copy number. A cut-off copy number of 1.6 is used to define pfpm2 multiple copy number.

Table 2

Piperaquine (PPQ) susceptibility (mean \pm SD)

\begin{tabular}{|lll|}
\hline Sample & PPQ-IC $_{50}(\mathrm{nM})$ & $\mathrm{PPQ}^{-I C_{90}}(\mathrm{nM})$ \\
\hline B5 & $112 \pm 54$ & $172 \pm 9$ \\
\hline B5-rexo-E415G-B8 & $185 \pm 54^{1}$ & $243 \pm 22^{1}$ \\
\hline ASAP-21 & $5,207 \pm 690^{2}$ & N.D. \\
\hline ASAP-168 & $111 \pm 17^{1}$ & $312 \pm 37^{1}$ \\
\hline
\end{tabular}

${ }^{1}$ No significant difference $(P$ value $>0.05)$ compared to B5 as tested by Mann-Whitney Test 
${ }^{2}$ Significant difference $(P$ value $=0.0167)$ between ASAP-21 and B5 as tested by Mann-Whitney Test

N.D. for not determined

\section{Drug sensitivity and survival assay of modified pfexo transgenic lines and clinical isolates}

The transgenic parasites expressing E415G mutant of pfexo and the clinical isolates were tested for their PPQ susceptibility (Table 2). No significant difference in $\mathrm{IC}_{50}$ values of B5-rexo-E415G-B8 and ASAP-168 parasites compared to B5 parasites was observed, while PPQ-IC 50 value of ASAP-21 parasites was significantly higher than that of B5 parasites. The same trend was observed for PPQ-IC ${ }_{90}$ values in that there was no significant difference in the $\mathrm{IC}_{90}$ values for B5-rexo-E415G-B8 and ASAP-168 parasites compared to B5 parasites. The PPQ-IC 90 of ASAP-21 exceeded the highest PPQ concentration used in this study, so the $\mathrm{IC}_{90}$ could not be reported.

To evaluate if the modified pfexo transgenic parasites and clinical isolates with PfEXO-E415G mutation have cross-resistance, parasite drug sensitivity was also assessed against a panel of antimalarial drugs (Table 3). No significant difference in IC 50 values between B5 and B5-rexo-

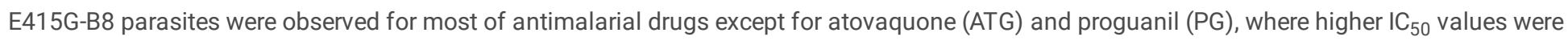
detected in comparison with the B5 parental line. The ASAP-21 parasite had significantly higher IC ${ }_{50}$ values for quinine (QN), chloroquine (CQ), atovaquone (ATQ) and doxycycline (DOX) but lower $\mathrm{IC}_{50}$ value for pyronaridine (PND), while the ASAP-168 parasite had reduced drug sensitivity against dihydroartemisinin (DHA), mefloquine (MQ), QN, and ATQ. The reduced drug sensitivity of QN and ATQ was observed in both ASAP-21 and ASAP-168 parasites.

To gain a better understanding of the ART and PPQ resistance phenotypes, the survival assay (RSA $\mathrm{A}_{0-3 \mathrm{~h}}$ and PSA 0 -3h) and PPQ-bimodal dose response curve were performed (Fig. 2). For RSA $\mathrm{R}_{0-3 \mathrm{~h}}$ (Fig. 2A), P. falciparum W2 was used as a control of ART-sensitive parasites, while IPC-4884 and IPC5202 parasites were controls for ART-resistant parasites. The ASAP-21 and ASAP-168 parasites, containing the PfK13-C580Y mutation, exhibited \% RSA survival rate of greater than 1, while B5 and B5-rexo-E415G-B8 parasites, carrying wild-type PfK13, exhibited \% RSA survival rate of less than 1, clearly validating the correlation between PfK13-C580Y mutation and ART resistance. The \% PSA survival rate of the modified pfexo transgenic lines and clinical isolates were next examined (Fig. 2B). PPQ-sensitive parasites P. falciparum W2, IPC-4884 and IPC-5202 were used as controls. The transgenic B5-rexo-E415G-B8 parasites harbouring merely PfEXO-E415G mutation had a \% PSA survival rate of less than 10 (a cut-off for PPQ resistance), similar to that of the parental B5 parasites. This suggested that the presence of PfEXO-E415G mutation alone could not confer the PPQ resistance phenotype. The ASAP-21 and ASAP-168 parasites, containing the PfEXO-E415G mutation in combination with either PfCRT mutation or multiple copies of pfpm2, exhibited a \% PSA survival rate higher than 10, indicative of PPQ-resistance. 
Table 3

In vitro susceptibility of $P$. falciparum, parental (B5), pfexo-modified (B5-rexo-E415G-B8) and adapted clinical parasites to dihydroartemisinin (DHA), artesunate (AS), mefloquine (MQ), quinine (QN), chloroquine (CQ), atovaquone (ATQ), lumefantrine (LUM), doxycycline (DOX), tafenoquine (TQ), cycloguanil (CYC), primaquine (PQ), proguanil (PG), and pyronaridine (PND). $($ mean \pm SD, nM)

\begin{tabular}{|llllll|}
\hline Drug & IC $_{50}(\mathrm{nM})$ & & & & \\
\cline { 2 - 5 } & B5 & B5-rexo-E415G-B8 & ASAP-21 & ASAP-168 & P-value \\
\hline DHA & $9 \pm 4$ & $14 \pm 6$ & $5 \pm 0.2$ & $2 \pm 1$ & $0.121^{3}$ \\
\hline AS & $7 \pm 5$ & $4 \pm 1$ & $3 \pm 0.4$ & $3 \pm 1$ & - \\
\hline MQ & $161 \pm 58$ & $82 \pm 37$ & $61 \pm 23$ & $50 \pm 20$ & $0.048^{3}$ \\
\hline QN & $546 \pm 180$ & $564 \pm 165$ & $71 \pm 3$ & $138 \pm 32$ & $0.017^{2,3}$ \\
\hline CQ & $562 \pm 218$ & $416 \pm 219$ & $64 \pm 12$ & $256 \pm 59$ & $0.017^{2}$ \\
\hline ATQ & $48 \pm 38$ & $114 \pm 1$ & $5 \pm 0.6$ & $4 \pm 1$ & $0.017^{1,2,3}$ \\
\hline LUM & $10 \pm 8$ & $4 \pm 1$ & $2 \pm 0.2$ & $3 \pm 1$ & - \\
\hline DOX & $13,523 \pm 2,337$ & $14,649 \pm 1,071$ & $8,527 \pm 349$ & $10,920 \pm 1,061$ & $0.024^{2}$ \\
\hline TQ & $263 \pm 143$ & $315 \pm 45$ & $189 \pm 13$ & $274 \pm 554$ & - \\
\hline CYC & $2,063 \pm 2,703$ & $705 \pm 15$ & $7,183 \pm 388$ & N.D. & - \\
\hline PQ & $3,094 \pm 2,015$ & $6,387 \pm 218$ & $5,720 \pm 1,160$ & $2,458 \pm 61$ & - \\
\hline PG & $12,522 \pm 7,247$ & $23,816 \pm 3,786$ & $2,114 \pm 243$ & $3,643 \pm 473$ & $0.033^{1}$ \\
\hline PND & $28 \pm 16$ & $25 \pm 4$ & $50 \pm 2$ & $12 \pm 6$ & $0.033^{2}$ \\
\hline
\end{tabular}

P-value calculated by Mann-Whitney Test

1, 2, 3 significant differences between data from B5-rexo-E415G-B8, ASAP-21, and ASAP-168 compared to B5 line, respectively.

N.D. for not determined

In addition to $\mathrm{PSA}_{0-3 \mathrm{~h}}, \mathrm{PPQ}$-resistant parasites have been reported to exhibit a bimodal dose-response curve with a second peak between 0.01 and $100 \mu \mathrm{M}$ and that the AUC correlates with the degree of PPQ resistance [16]. B5 and B5-rexo-E415G-B8 parasites did not exhibit the bimodal dose-response curve, confirming the PPQ-sensitive phenotype, whereas both ASAP-21 and ASAP-168 parasites clearly showed the bimodal doseresponse curve with the AUC of 1029 and 596, respectively (Figs. 3C-F). The AUC of these parasites was in good agreement with both IC ${ }_{50}$ and $\mathrm{IC}_{90}$ values in that ASAP-21 parasites are more resistant to PPQ than ASAP-168 parasites.

\section{Growth Assay}

To assess whether the introduction of E415G mutation into PfEXO affects the parasite fitness, growth assays of B5-rexo-E415G-B8 parasites was carried out in comparison with W2, 3D7, B5 and the other two culture adapted clinical isolates (ASAP-21 and ASAP-168) (Fig. 3). Parasite lines originating from Cambodia (i.e., B5, B5-rexo-E415G-B8, ASAP-21 and ASAP-168) grew much more slowly than W2 (an Indochina clone exhibiting $\mathrm{CQ}$ resistance) and 37D (African origin) lines. Parasite B5-rexo-E415G-B8, harbouring pfexo-E415G mutation, seemed to grow slightly faster than its parental line B5, suggesting this mutation may improve the parasite's fitness. In the presence of PfK13-C580Y, PfEXO-E415G, novel PfCRTF145I mutations and multiple copy number of pfpm2, ASAP-21 parasite had a severely retarded growth rate. ASAP-168, having the similar genetic background to ASAP-21 but without the PfCRT F145I mutation, grew marginally better than ASAP-21 parasites. This observation implies an important contribution of the strain background toward parasite fitness, especially when PfK13-C580Y, PfCRT F145I mutations and multiple copy number of pfpm2 were present.

\section{Discussion}

Validated molecular markers, such as pfk13 for ART resistance, have been widely employed as identification and prediction tools for the emergence of drug-resistant $P$. falciparum [50]. While a molecular marker of PPQ resistant $P$. falciparum malaria has been reported [42], there is 
still a pressing need for validating potential new or additive molecular markers to determine effects on PPQ as a partner drug in ACT. In this study, it is evident that the $\mathrm{E} 415 \mathrm{G}$ mutation in a gene encoding exonuclease cannot alone reduce PPQ susceptibility nor improve parasite survival rates that are characteristic of PPQ resistant isolates circulating in the Greater Mekong Subregion [17]

Exonucleases are essential to genome stability, catalysing the removal of a single nucleotide monophosphate (dNMP) from the end of one strand of DNA and acting as a proof-reader during DNA replication [51]. Exonucleases are highly conserved and can be classified into families based on both sequence and functional homology, including the 5'-3' exo C-terminal domain (CTD) superfamily, the RNAaseH domain superfamily i.e., DnaQ-like family and other $3^{\prime}-5^{\prime}$ exonucleases. The pfexo gene from P. falciparum (PF3D7_1362500) encodes a protein with a mass of 86.7 kDa with a 3'-5' exonuclease domain at the C-terminus. In this study, the P. falciparum B5 line used for genome editing had one mutation (K614N) in exonuclease). Nucleases may be partially or fully redundant, depending on the pathway, and such redundancy might complement functional losses. Zhang Q et. al. [52] used bioinformatics to predict eight putative RNA exosome-associated proteins in the $P$. falciparum genome, including exoribonuclease functional domain-containing proteins Dis3 and Rrp6, the latter of which was found in PfEXO of this study. Even though, the genuine function of PfEXO has not been revealed, it is clear that the E415G mutation in PfEXO is unlikely to impact on the catalytic activity of PfEXO.

CRISPR/Cas9 approaches have transformed the speed and scale with which Plasmodium genome editing can be achieved, and the approach has been employed to introduce point mutations into several $P$. falciparum genes [53]. In this study, instead of using $P$. falciparum 3D7 or Dd2 lines, the Cambodia adapted-B5 line [24] was employed with the hypothesis that the B5 line has a close genetic background to the currently circulating P. falciparum in Cambodia where both ART- and PPQ-resistance parasites have been detected. It has been previously shown that different phenotypes were observed when using different parasite backgrounds. Targeted gene disruption of either pfpm2 or pfpm3 in the 3D7 genetic background caused a only slight decrease in PPQ susceptibility [54] and pfpm2 and pfpm3 overexpression in 3D7 did not alter the sensitivity of $P$. falciparum to PPQ [55]. However, when P. falciparum Dd2 parasites with copy number variation in pfpm2 were generated [29], pfpm2 amplification contributed to PPQ resistance, with a bimodal dose-response observed. In addition to a qPCR assay to detect $p f p m 2$ copy number variation, the breakpoint assay, as described by Imwong M, et. al. [56], was carried out. It was found that in parasites with a pfpm2/3 copy number above the cut-off of $1.52,88 \%$ were confirmed to have pfpm $2 / 3$ amplification by the breakpoint assay, while for those $<1.14$ (the cut-off for a single copy number) and for an intermediate value between 1.14-1.52, the proportion of pfpm $2 / 3$ amplified parasites using the breakpoint SNP were $4 \%$ and $38 \%$, respectively. In this study, the ASAP-168 parasite was found to have pfpm 2 copy number variation of 1.11 , lower than the cut-off for a single copy number, but the breakpoint assay confirmed the gene duplication of pfpm2. Therefore, it is important to also apply the breakpoint assay to isolates with copy number values $<1.52$, to capture all isolates with pfpm2/3 amplification.

$\mathrm{IC}_{90}$ values, $\mathrm{PPQ}$ survival rates, and bimodal dose-response curves were used for assessing PPQ resistance in vitro [16, 17, 48]. All three assays confirmed that the engineered parasites harboring the E415G-PfEXO mutation (B5-rexo-E415G-B8) did not show a PPQ resistant phenotype. On the contrary, parasites with the combination of PfEXO-E415G mutation with either novel PfCRT or pfpm2 multiple copies demonstrated reduced $\mathrm{IC}_{90}$ susceptibility, high PPQ survival rates, and a second peak of bimodal curve (Fig. 3). If the PfEXO-E415G mutation does not alter the PPQ susceptibility, this finding also recapitulates the work of Silva, M et. al. [29] that parasites with the pfpm2 multiple copy alone (i.e. ASAP-168) show reduced PPQ susceptibility and involved in PPQ resistance. Nonetheless, when the novel PfCRT was added on top of the pfpm2 multiple copies, the level of PPQ resistance increased tremendously as judged by the $\mathrm{IC}_{50}$ and $\mathrm{IC}_{90}$ values as well as AUC between $0.01-100 \mu \mathrm{M}$ of PPQ in the bimodal dose-response curve. Bopp, S et. al. [16] showed that when exposed up to $10 \mu \mathrm{M}$ PPQ for 12 hours, PPQ resistance parasites could survive and complete their lifecycle. It was also previously evident that the introduction of novel PfCRT mutations resulted in a fitness cost for the mutations. Similar trends were observed in this study; parasites holding either novel PfCRT or pfpm2 multiple copies grew much slower than those without mutations. It is likely that introducing PfEXO-E415G mutations may account for better fitness compared to parasites with the same genetic background. Even though the scope of this work could not address the function of PfEXO, it is evidently showed that the E415G mutation in PfEXO does not alter PPQ susceptivity, but rather affects parasite fitness.

\section{Conclusion}

In summary, this study suggests that the E415G mutation in PfEXO may not influence PPQ susceptibility and that caution should be used if assessing this mutation as a molecular marker to monitor drug resistance. The insertion of the PfEXO-E415G mutation did not lead to an increased PPQ- $\mathrm{IC}_{90}$ or improve \% PPQ survival, suggesting that this mutation is not associated with PPQ resistance and unsuitable to be used as a molecular marker for monitoring PPQ-resistant parasites. Additionally, this specific mutation resulted in parasites that grew better than those with the same background, highlighting the importance of genetic mutations toward parasite fitness and resistance.

\section{Declarations}

\section{Acknowledgements}


The following reagents were obtained through BEI Resources, NIAID, NIH: Plasmodium falicaprum, Strain IPC-4884 (MRA-1238) and IPC-5202 (MRA-1240), contributed by Didier Ménard. We would like to thank Professor Michael J Blackman (The Francis Crick Institute, UK) for providing the pDC2-Cas9-hDHFR-yFCU plasmid as well as insightful comments and suggestions, Dr. Ellen Knuepfer (The Royal Veterinary College, UK) for PkpSKIP_Pk47 plasmid, and Dr. Michele D. Spring (USAMD-AFRIMS) for valuable comments and suggestions. The authors would like to thank the clinical and laboratory staff from Molecular and Immunology section at AFRIMS-BPD who made the study possible.

\section{Author's contributions}

NB conceived, designed, and executed the study. BAV, MW, JSG, and NCW conceived, and supported the study. NB constructed all plasmids used in this study. NB and KK performed transfection, cloning and selection processes. KK, CT and CP performed cell culture, drug susceptibility assay, survival assay. CP performed cell growth assay and flow cytometry analysis. NB wrote the first draft. All authors edited, reviewed, and approved the final manuscript.

\section{Disclaimer}

Material has been reviewed by the Walter Reed Army Institute of Research. There is no objection to its presentation/publication. The opinions or assertions contained herein are the private views of the author, and are not to be construed as official, or as reflecting true views of the Department of the Army or the Department of Defense. The investigators have adhered to the policies for protection of human subjects as prescribed in AR 70-25.

\section{Ethics approval and consent to participate}

Not applicable

\section{Consent for publication}

Not applicable

\section{Availability of data and materials}

All data generated or analysed during this study are included in this published article and its supplementary information files.

\section{Competing Interests}

The authors declare that they have no competing interests.

\section{Funding}

This work was supported by Defense Malaria Assistance Program (DMAP). The funding source had no role in the analysis or interpretation of data, preparation of the manuscript or the decision to publish.

\section{References}

1. World Malaria Report. Geneva: World Health Organization; 2019.

2. Noedl H, Se Y, Schaecher K, Smith BL, Socheat D, Fukuda MM, et al. Evidence of artemisinin-resistant malaria in western Cambodia. N Engl J Med. 2008;359:2619-20.

3. Dondorp AM, Nosten F, Yi P, Das D, Phyo AP, Tarning J, et al. Artemisinin resistance in Plasmodium falciparum malaria. N Engl J Med. 2009;361:455-67.

4. Amaratunga C, Sreng S, Suon S, Phelps ES, Stepniewska K, Lim P, et al. Artemisinin-resistant Plasmodium falciparum in Pursat province, western Cambodia: a parasite clearance rate study. Lancet Infect Dis. 2012;12:851-8.

5. WHO. Guidelines fro the treatment of malaria. 3rd edition. Geneva: World Health Organization Press; 2015.

6. Saunders DL, Vanachayangkul P, Lon C, Program USAMMR, National Center for Parasitology E, Malaria C, et al. Dihydroartemisininpiperaquine failure in Cambodia. N Engl J Med. 2014;371:484-5.

7. Leang R, Taylor WR, Bouth DM, Song L, Tarning J, Char MC, et al. Evidence of Plasmodium falciparum Malaria Multidrug Resistance to Artemisinin and Piperaquine in Western Cambodia: Dihydroartemisinin-Piperaquine Open-Label Multicenter Clinical Assessment. Antimicrob Agents Chemother. 2015;59:4719-26.

8. Spring MD, Lin JT, Manning JE, Vanachayangkul P, Somethy S, Bun R, et al. Dihydroartemisinin-piperaquine failure associated with a triple mutant including kelch13 C580Y in Cambodia: an observational cohort study. Lancet Infect Dis. 2015;15:683-91. 
9. Amaratunga C, Lim P, Suon S, Sreng S, Mao S, Sopha C, et al. Dihydroartemisinin-piperaquine resistance in Plasmodium falciparum malaria in Cambodia: a multisite prospective cohort study. Lancet Infect Dis. 2016;16:357-65.

10. Phuc BQ, Rasmussen C, Duong TT, Dong LT, Loi MA, Menard D, et al. Treatment Failure of Dihydroartemisinin/Piperaquine for Plasmodium falciparum Malaria, Vietnam. Emerg Infect Dis. 2017;23:715-7.

11. van der Pluijm RW, Imwong M, Chau NH, Hoa NT, Thuy-Nhien NT, Thanh NV, et al. Determinants of dihydroartemisinin-piperaquine treatment failure in Plasmodium falciparum malaria in Cambodia, Thailand, and Vietnam: a prospective clinical, pharmacological, and genetic study. Lancet Infect Dis. 2019;19:952-61.

12. Witkowski B, Amaratunga C, Khim N, Sreng S, Chim P, Kim S, et al. Novel phenotypic assays for the detection of artemisinin-resistant Plasmodium falciparum malaria in Cambodia: in-vitro and ex-vivo drug-response studies. Lancet Infect Dis. 2013;13:1043-9.

13. Amato R, Lim P, Miotto O, Amaratunga C, Dek D, Pearson RD, et al. Genetic markers associated with dihydroartemisinin-piperaquine failure in Plasmodium falciparum malaria in Cambodia: a genotype-phenotype association study. Lancet Infect Dis. 2017;17:164-73.

14. Parobek CM, Parr JB, Brazeau NF, Lon C, Chaorattanakawee S, Gosi P, et al. Partner-Drug Resistance and Population Substructuring of Artemisinin-Resistant Plasmodium falciparum in Cambodia. Genome Biol Evol. 2017;9:1673-86.

15. Witkowski B, Duru V, Khim N, Ross LS, Saintpierre B, Beghain J, et al. A surrogate marker of piperaquine-resistant Plasmodium falciparum malaria: a phenotype-genotype association study. Lancet Infect Dis. 2017;17:174-83.

16. Bopp S, Magistrado P, Wong W, Schaffner SF, Mukherjee A, Lim P, et al. Plasmepsin II-III copy number accounts for bimodal piperaquine resistance among Cambodian Plasmodium falciparum. Nat Commun. 2018;9:1769.

17. Duru V, Khim N, Leang R, Kim S, Domergue A, Kloeung N, et al. Plasmodium falciparum dihydroartemisinin-piperaquine failures in Cambodia are associated with mutant $\mathrm{K} 13$ parasites presenting high survival rates in novel piperaquine in vitro assays: retrospective and prospective investigations. BMC Med. 2015;13:305.

18. Agrawal S, Moser KA, Morton L, Cummings MP, Parihar A, Dwivedi A, et al. Association of a Novel Mutation in the Plasmodium falciparum Chloroquine Resistance Transporter With Decreased Piperaquine Sensitivity. J Infect Dis. 2017;216:468-76.

19. Ross LS, Dhingra SK, Mok S, Yeo T, Wicht KJ, Kumpornsin K, et al. Emerging Southeast Asian PfCRT mutations confer Plasmodium falciparum resistance to the first-line antimalarial piperaquine. Nat Commun. 2018;9:3314.

20. Dhingra SK, Small-Saunders JL, Menard D, Fidock DA. Plasmodium falciparum resistance to piperaquine driven by PfCRT. Lancet Infect Dis. 2019;19:1168-9.

21. Thanh NV, Thuy-Nhien N, Tuyen NT, Tong NT, Nha-Ca NT, Dong LT, et al. Rapid decline in the susceptibility of Plasmodium falciparum to dihydroartemisinin-piperaquine in the south of Vietnam. Malar J. 2017;16:27.

22. Diakite SAS, Traore K, Sanogo I, Clark TG, Campino S, Sangare M, et al. A comprehensive analysis of drug resistance molecular markers and Plasmodium falciparum genetic diversity in two malaria endemic sites in Mali. Malar J. 2019;18:361.

23. Robert MG, Tsombeng FF, Gendrot M, Diawara S, Madamet M, Kounta MB, et al. Baseline Ex Vivo and Molecular Responses of Plasmodium faciparum isolates to Piperaquine before Implementation of Dihydroartemisinin-Piperaquine in Senegal. Antimicrob Agents Chemother. 2019.

24. Boonyalai N, Vesely BA, Thamnurak C, Praditpol C, Fagnark W, Kirativanich K, et al. Piperaquine resistant Cambodian Plasmodium falciparum clinical isolates: in vitro genotypic and phenotypic characterization. Malar J. 2020;19:269.

25. Quang HH, Chavchich M, Trinh NTM, Edgel KA, Edstein MD, Martin NJ. Multidrug-Resistant Plasmodium falciparum Parasites in the Central Highlands of Vietnam Jeopardize Malaria Control and Elimination Strategies. Antimicrob Agents Chemother. $2021 ; 65$.

26. Si Y, Zeng W, Li N, Wang C, Siddiqui F, Zhang J, et al. In Vitro Susceptibility of Plasmodium falciparum Isolates from the China-Myanmar Border Area to Piperaquine and Association with Candidate Markers. Antimicrob Agents Chemother. 2021.

27. Phong NC, Chavchich M, Quang HH, San NN, Birrell GW, Chuang I, et al. Susceptibility of Plasmodium falciparum to artemisinins and Plasmodium vivax to chloroquine in Phuoc Chien Commune, Ninh Thuan Province, south-central Vietnam. Malar J. 2019;18:10.

28. Hussien M, Abdel Hamid MM, Elamin EA, Hassan AO, Elaagip AH, Salama AHA, et al. Antimalarial drug resistance molecular makers of Plasmodium falciparum isolates from Sudan during 2015-2017. PLoS One. 2020;15:e0235401.

29. Silva M, Calcada C, Teixeira M, Veiga MI, Ferreira PE. Multigenic architecture of piperaquine resistance trait in Plasmodium falciparum. Lancet Infect Dis. 2020;20:26-7.

30. Madeira F, Park YM, Lee J, Buso N, Gur T, Madhusoodanan N, et al. The EMBL-EBI search and sequence analysis tools APIs in 2019. Nucleic Acids Res. 2019;47:W636-41.

31. Almagro Armenteros JJ, Tsirigos KD, Sonderby CK, Petersen TN, Winther O, Brunak S, et al. SignalP 5.0 improves signal peptide predictions using deep neural networks. Nat Biotechnol. 2019;37:420-3.

32. Blum M, Chang HY, Chuguransky S, Grego T, Kandasaamy S, Mitchell A, et al. The InterPro protein families and domains database: 20 years on. Nucleic Acids Res. 2021;49:D344-54. 
33. Trager W, Jensen JB. Human malaria parasites in continuous culture. Science. 1976;193:673-5.

34. Saul A, Myler P, Elliott T, Kidson C. Purification of mature schizonts of Plasmodium falciparum on colloidal silica gradients. Bull World Health Organ. 1982;60:755-9.

35. Harris PK, Yeoh S, Dluzewski AR, O'Donnell RA, Withers-Martinez C, Hackett F, et al. Molecular identification of a malaria merozoite surface sheddase. PLoS Pathog. 2005;1:241-51.

36. Lambros C, Vanderberg JP. Synchronization of Plasmodium falciparum erythrocytic stages in culture. J Parasitol. 1979;65:418-20.

37. Knuepfer E, Napiorkowska M, van Ooij C, Holder AA. Generating conditional gene knockouts in Plasmodium - a toolkit to produce stable DiCre recombinase-expressing parasite lines using CRISPR/Cas9. Sci Rep. 2017;7:3881.

38. Knuepfer E, Wright KE, Kumar Prajapati S, Rawlinson TA, Mohring F, Koch M, et al. Divergent roles for the RH5 complex components, CyRPA and RIPR in human-infective malaria parasites. PLoS Pathog. 2019;15:e1007809.

39. Collins CR, Das S, Wong EH, Andenmatten N, Stallmach R, Hackett F, et al. Robust inducible Cre recombinase activity in the human malaria parasite Plasmodium falciparum enables efficient gene deletion within a single asexual erythrocytic growth cycle. Mol Microbiol. 2013;88:687-701.

40. Janse CJ, Franke-Fayard B, Mair GR, Ramesar J, Thiel C, Engelmann S, et al. High efficiency transfection of Plasmodium berghei facilitates novel selection procedures. Mol Biochem Parasitol. 2006;145:60-70.

41. Thomas JA, Collins CR, Das S, Hackett F, Graindorge A, Bell D, et al. Development and Application of a Simple Plaque Assay for the Human Malaria Parasite Plasmodium falciparum. PLoS One. 2016;11:e0157873.

42. Ariey F, Witkowski B, Amaratunga C, Beghain J, Langlois AC, Khim N, et al. A molecular marker of artemisinin-resistant Plasmodium falciparum malaria. Nature. 2014;505:50-5.

43. Talundzic E, Okoth SA, Congpuong K, Plucinski MM, Morton L, Goldman IF, et al. Selection and spread of artemisinin-resistant alleles in Thailand prior to the global artemisinin resistance containment campaign. PLoS Pathog. 2015;11:e1004789.

44. Pelleau S, Moss EL, Dhingra SK, Volney B, Casteras J, Gabryszewski SJ, et al. Adaptive evolution of malaria parasites in French Guiana: Reversal of chloroquine resistance by acquisition of a mutation in pfcrt. Proc Natl Acad Sci U S A. 2015;112:11672-7.

45. Ansbro MR, Jacob CG, Amato R, Kekre M, Amaratunga C, Sreng S, et al. Development of copy number assays for detection and surveillance of piperaquine resistance associated plasmepsin 2/3 copy number variation in Plasmodium falciparum. Malar J. 2020;19:181.

46. Chaorattanakawee S, Tyner SD, Lon C, Yingyuen K, Ruttvisutinunt W, Sundrakes S, et al. Direct comparison of the histidine-rich protein-2 enzyme-linked immunosorbent assay (HRP-2 ELISA) and malaria SYBR green I fluorescence (MSF) drug sensitivity tests in Plasmodium falciparum reference clones and fresh ex vivo field isolates from Cambodia. Malar J. 2013;12:239.

47. Chaorattanakawee S, Saunders DL, Sea D, Chanarat N, Yingyuen K, Sundrakes S, et al. Ex Vivo Drug Susceptibility Testing and Molecular Profiling of Clinical Plasmodium falciparum Isolates from Cambodia from 2008 to 2013 Suggest Emerging Piperaquine Resistance. Antimicrob Agents Chemother. 2015;59:4631-43.

48. Chaorattanakawee S, Lon C, Jongsakul K, Gawee J, Sok S, Sundrakes S, et al. Ex vivo piperaquine resistance developed rapidly in Plasmodium falciparum isolates in northern Cambodia compared to Thailand. Malar J. 2016;15:519.

49. Wojnarski M, Lon C, Vanachayangkul P, Gosi P, Sok S, Rachmat A, et al. Atovaquone-Proguanil in Combination With Artesunate to Treat Multidrug-Resistant P. falciparum Malaria in Cambodia: An Open-Label Randomized Trial. Open Forum Infect Dis. $2019 ; 6:$ ofz314.

50. Plowe CV, Roper C, Barnwell JW, Happi CT, Joshi HH, Mbacham W, et al. World Antimalarial Resistance Network (WARN) III: molecular markers for drug resistant malaria. Malar J. 2007;6:121.

51. Mason PA, Cox LS. The role of DNA exonucleases in protecting genome stability and their impact on ageing. Age (Dordr). 2012;34:1317-40.

52. Zhang Q, Siegel TN, Martins RM, Wang F, Cao J, Gao Q, et al. Exonuclease-mediated degradation of nascent RNA silences genes linked to severe malaria. Nature. 2014;513:431-5.

53. Lee MCS, Lindner SE, Lopez-Rubio JJ, Llinas M. Cutting back malaria: CRISPR/Cas9 genome editing of Plasmodium. Brief Funct Genomics. 2019;18:281-9.

54. Mukherjee A, Gagnon D, Wirth DF, Richard D. Inactivation of Plasmepsins 2 and 3 Sensitizes Plasmodium falciparum to the Antimalarial Drug Piperaquine. Antimicrob Agents Chemother. 2018; 62.

55. Loesbanluechai D, Kotanan N, de Cozar C, Kochakarn T, Ansbro MR, Chotivanich K, et al. Overexpression of plasmepsin II and plasmepsin III does not directly cause reduction in Plasmodium falciparum sensitivity to artesunate, chloroquine and piperaquine. Int $\mathrm{J}$ Parasitol Drugs Drug Resist. 2018;9:16-22.

56. Imwong M, Suwannasin K, Srisutham S, Vongpromek R, Promnarate C, Saejeng A, et al. Evolution of multidrug resistance in Plasmodium falciparum: a longitudinal study of genetic resistance markers in the Greater Mekong Subregion. Antimicrob Agents Chemother. 2021:AAC0112121. 
Figures

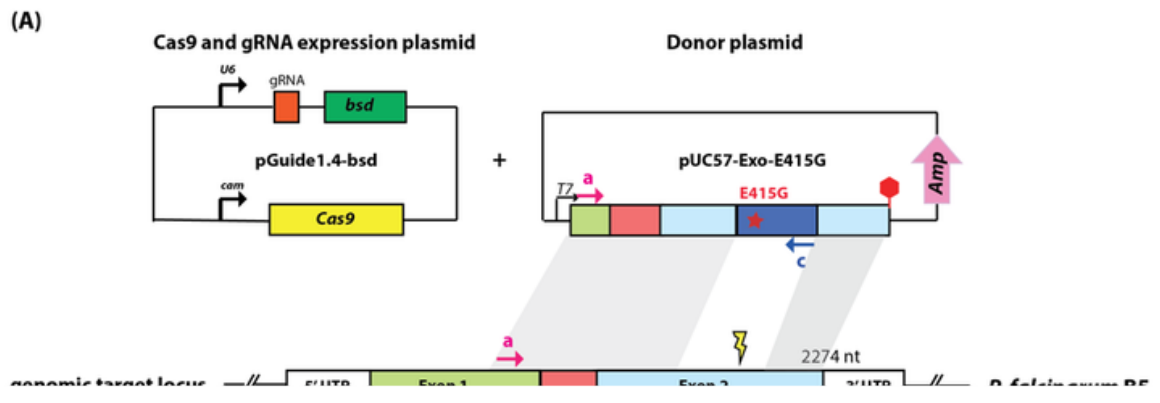

(C)

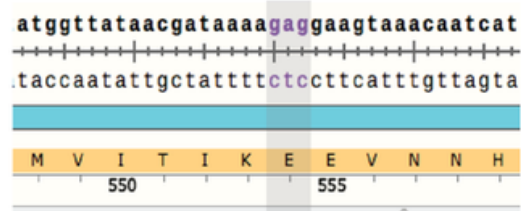

\section{Figure 1}

Gene editing of pfexo. (A) CRISPR-Cas9 editing was used to install the pfex ${ }^{E 415 G}$ variant codon into the endogenous locus. Using a donor plasmid and Cas9-aided homologous recombination, the region of the pfexo encoding Met409 to Glu514 was replaced with a recodonized pfexo gene (navy blue) with the point mutation at residue 415 (a red star) from Glu to Gly. Stop codon is represented as a red hexagon. Position of hybridisation of primers used for confirmation of the integration event by diagnostic PCR are shown as coloured arrows. A schematic of the cotransfected plasmid for expression of Cas9 nuclease and the guide RNAs is also shown. (B) Diagnostic PCR analysis of genomic DNA of the control parental B5 line, the transfected parasites prior to limiting dilution cloning and clone parasite lines expressing pfex ${ }^{E 415 G}$ (clone B8). Expected sizes of the various PCR amplicons are indicated on the right of the gel, whilst the left-hand lane contains double-stranded DNA ladder marker fragment (Quick-load Purple $1 \mathrm{~kb}$ Plus DNA ladder (New England Biolabs)). (C) Chromatograms of a region of exon 2 obtained from the PCR product amplified pfexo gene of B5 and B5-rexo-E415G-B8 lines. The codon "GAG" encoding Glu in B5 parasites is changed to "GGC" encoding Gly in B5-rexo-E415G-B8 clone. 

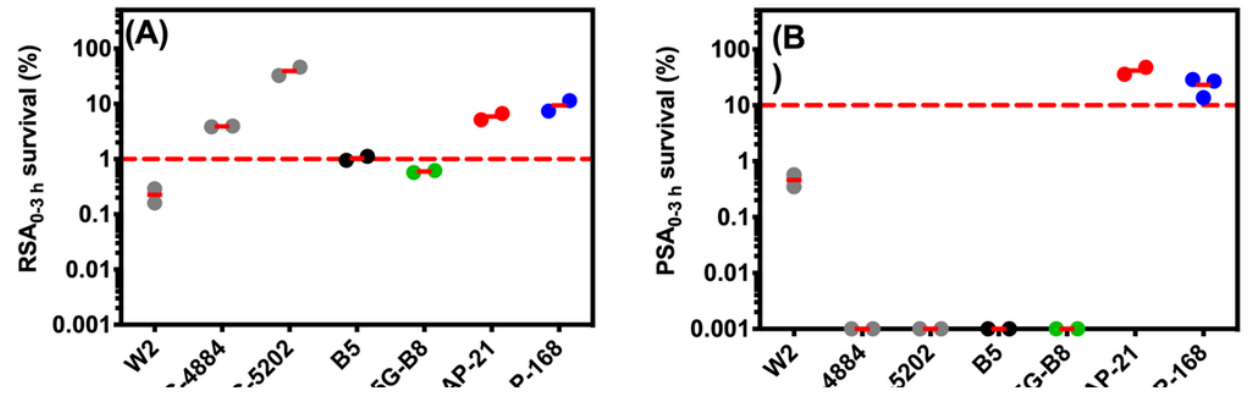

Figure 2

Phenotypic analysis of pfexo transgenic lines and clinical isolates. (A-B) In vitro $\mathrm{RSA}_{0-3 \mathrm{~h}}$ and $\mathrm{PSA}_{0-3 \mathrm{~h}}$ survival rates, respectively, for standard laboratory-adapted clones (W2 for an ART-sensitive control, IPC-4884 and IPC-5202 for ART-resistance control), B5 (parental line, B5-rexo-E415GB8 (modified pfexo transgenic line) and culture-adapted clinical isolates (ASAP-21 and ASAP-168). The dashed line represents the 1\% survival rate cut-off that differentiates ART-resistance ( $\geq 1 \%)$ from ART-sensitive $(<1 \%)$ parasites in RSAs or the 10\% survival rate cut-off that distinguishes PPQ-resistance ( $\geq 10 \%)$ from PPQ-sensitive (< 10\%) parasites in PSAs. Two independent biological replicates were performed. Zero values of \% survival rate were plotted as $0.001 \%$ in logarithmic scale. (C-F) PPQ-bimodal dose-response curves of B5, B8, IPC-4884, and IPC-5202, respectively. Increasing the starting concentration and number of data points (24 points) for HRP2 ELISA dose-response curve provided a bimodal distribution of parasite response to PPQ exposure for PPQ-resistant parasites. ASAP-21 and ASAP-168 shows a second peak in PPQ concentration $0.01-100 \mu \mathrm{M}$. The concentration range used to calculate the area under the curve (AUC) is indicated. Data are shown as mean values from three biological replicates with S.D. 


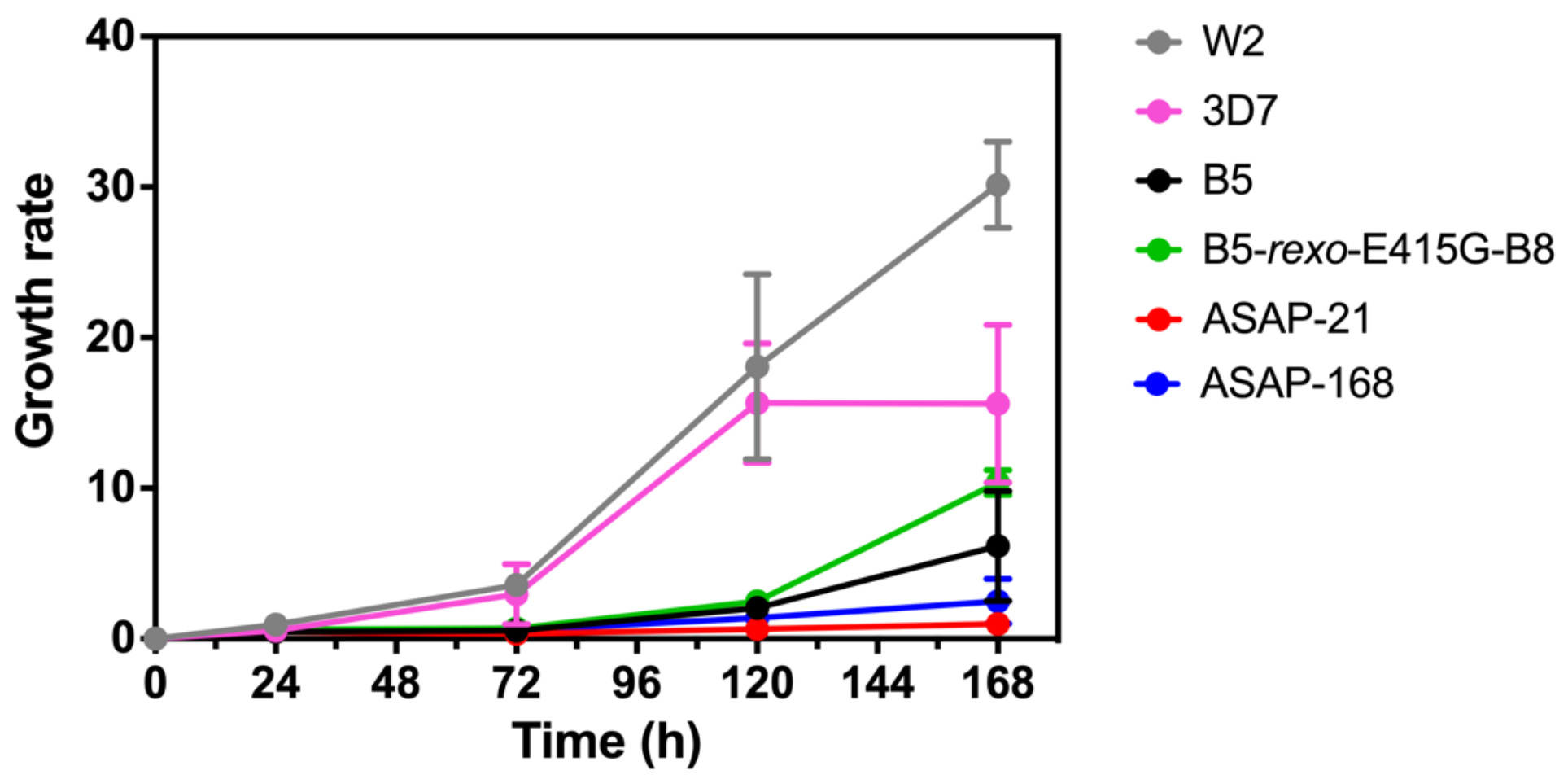

Figure 3

Analysis of growth rates. P. falciparum parasites with PfEXO wild-type (W2, 3D7 and B5), PfEXO-E415G transgenic line (B5-rexo-E415G-B8), and clinical isolates with the combination of PfEXO-E415G and multiple pfpm 2 copies (ASAP-21 and ASAP-168) were synchronized (initiated at $0.1 \%$ parasitaemia ring stage, $2 \%$ haematocrit) and followed for 168 hours. Nucleic acids were stained with SYBR Green I and parasitaemia assessed by flow cytometry. Raw values were corrected, and data represent mean \pm S.D. from 3 independent experiments.

\section{Supplementary Files}

This is a list of supplementary files associated with this preprint. Click to download.

- 20220104SupportingInfo.docx 